\title{
ESTRÉS Y VIOLENCIA DOMESTICA: UN ESTUDIO EN ADULTOS REFERENTES DE NIÑOS, NIÑAS Y ADOLESCENTES
}

\section{STRESS AND DOMESTIC VIOLENCE: A STUDY IN SIGNIFICANT ADULTS TO CHILDREN AND ADOLESCENTS}

\author{
Alvaro Capano \\ Universidad Católica del Uruguay \\ Asociación Civil SOMOS*, Uruguay \\ Ayrel Pacheco \\ Universidad de la República, Uruguay \\ Asociación Civil SOMOS, Uruguay
}

\begin{abstract}
Resumen: Este artículo reporta la investigación-acción realizada con 60 adultos referentes (padres, madres, cuidadores, etc.) de niños/as y adolescentes que han vivido situaciones de violencia doméstica en alguna de sus formas (maltrato y/o abuso sexual). El estudio de carácter descriptivo tiene como objetivo identificar y cuantificar los eventos vitales estresantes y la percepción del mismo; a partir de los resultados del Cuestionario de Estrés Percibido -CEP - (Cohen, Kamarck y Mermelstein, 1983; Gracia, Herrero y Musitu, 2002) y el Listado de Eventos Vitales Estresantes - LEE - (Gracia, Herrero y Musito, 2002). Resultados: el $30,5 \%$ experimentaron entre 5 y 10 eventos vitales estresantes; el 10,2\% más de 10 . De la categorización realizada, cinco de los ítems seleccionados por los adultos refieren a dificultades vinculares/relacionales, dos a económicas/laborales y una a dificultades por problemas generales/asuntos sin resolver. En la puntuación sobre percepción de falta de control, un $45 \%$ contesta "bastante y muy a menudo" mientras que "a veces" alcanza un $30 \%$; la puntuación sobre sentimientos de control alcanza un $11 \%$ en "nunca y casi nunca" mientras que "a veces" llega a un 33\%. En conclusión los adultos referentes informan haber padecido una cantidad significativa de eventos vitales estresantes y plantean niveles de percepción de estrés elevados.
\end{abstract}

Palabras Clave: estrés, violencia doméstica, abuso infantil

\begin{abstract}
The article deals with the action research carried out with 60 significant adults (fathers, mothers, care giver, etc.) for children and adolescents who have experienced any kind of domestic violence (child sexual abuse or maltreatment). This descriptive research aims to identify and assess stressful life events and their perception from the results of the Perceived Stress Questionnaire -PSQ- (Cohen, Kamarck \& Mermelstein, 1983; Gracia, Herrero \& Musitu, 2002) and the Stressful Life Events List (Gracia, Herrero \& Musitu, 2002). Results: 30.5 percent suffered between 5 and 10 stressful life events; 10.2 percent suffered more than 10 of these events. Of our selection, five of the items selected by adults refer to difficulties in family ties and relationships, two refer to financial or employment issues, and one refers to general/unresolved issues. In the score related to the perception of lack of control, 45 percent answered "quite often" while "sometimes" reached 30 percent. Regarding a feeling of control, 11 percent answered "never and almost never" while "sometimes" reached 33 percent. In conclusion, significant adults report to have suffered an important amount of stressful life events and show high levels of stress perception.
\end{abstract}

Keywords: stress, domestic violence, child abuse

\section{Introducción}

La violencia doméstica constituye un problema en primer término de derechos humanos; trasciende la intimidad o privacidad del ámbito familiar y se constituye además en un problema social y sanitario, público y por ende en una responsabilidad colectiva, tanto en su impacto hacia las mujeres como hacia niños, niñas y adolescentes, en adelante NNA. En Uruguay la violencia doméstica es el delito más denunciado ante la policía luego de los hurtos, superando los delitos por rapiña (Observatorio Nacional sobre Violencia y Criminalidad, 2012).
¿Qué entendemos por violencia doméstica? La violencia doméstica, en adelante VD es:

... toda acción u omisión, directa o indirecta, que por cualquier medio menoscabe, limitando ilegítimamente el libre ejercicio o goce de los derechos humanos de una persona, causada por otra, con la cual tenga o haya tenido una relación de noviazgo o con la cual tenga o haya tenido una relación

*SOMOS: Asociación Civil de asistencia psicosocial y jurídica en abuso y maltrato, de Montevideo-Uruguay. 
afectiva basada en la cohabitación y originada por parentesco, por matrimonio o por unión de hecho (Ley 17514 , 2002, p. 1).

La VD abarcaría tanto la violencia de género como la generacional. Hay diferentes tipos de VD, entre los miembros de la pareja, entre los hijos, entre padres e hijos, etc (Sanmartin, 2006).

En relación a la violencia y los NNA, un estudio llevado adelante por De los Campos, Solari y González (2008), tuvo como objetivo medir conductas violentas exclusivamente en los adultos y refieren los autores que, si tomamos en cuenta a NNA de 0 a 17 años, se observan prácticas violentas, psicológicas y físicas, en el $80 \%$ de los adultos entrevistados. Si incluimos la negligencia como una conducta violenta y consideramos de forma exclusiva a los adultos a cargo de NNA el porcentaje asciende al $86 \%$. En cuanto a la violencia física en sus diversas manifestaciones, cabe consignar que fue reportada por el $55 \%$ de los adultos entrevistados.

Las familias de NNA que llegan a los servicios de atención han vivido situaciones de violencia doméstica, específicamente maltrato, abuso sexual y/o explotación sexual comercial; son familias que se caracterizan por la polisintomatología y crisis recurrentes, la desorganización, el abandono de las funciones parentales, el aislamiento (Gómez, Muñoz y Haz, 2007) y la multiasistencia sostenida en el tiempo de la historia familiar.

La multiplicidad de factores que operan tanto desde adentro como desde afuera del sistema familiar (Palacios, Jiménez, Oliva y Saldaña, 1998), trae aparejado el incremento de los conflictos y son fuentes de estrés que agotan los recursos y la capacidad de la familia para resolver y responder adecuadamente a las dificultades (Navarro, Musitu y Herrero, 2007).

El impacto y la acumulación de problemas, al pasar cierto nivel, saturan los mecanismos homeostáticos de las familias y los individuos, imposibilitando contar con el equilibrio necesario. Crea un círculo negativo de dificultades en aumento, que conducen a permanentes crisis y a un deterioro de la calidad de vida de las familias (Pakman, 2006). A estas familias algunos autores como Linares (1997); Pakman (2006); las Ilaman multiproblemáticas, otros autores como, Minuchin, Colapinto y Minuchin (2000), las denominan familias desorganizadas. Estas familias además de contar con múltiples proble- mas, atravesar crisis, tensiones, padecer patologías individuales, familiares o sociales, tienen una importante falta de recursos, redes sociales y apoyo social, volviéndolas familias muy vulnerables y con un alto riesgo psicosocial. Las familias en riesgo psicosocial, son aquellas en las que las personas a cargo del cuidado, atención y educación de NNA, por circunstancias personales, relacionales y/o contextuales hacen dejación de sus funciones parentales o hacen un uso inadecuado de las mismas, comprometiendo o perjudicando el desarrollo personal y social de estos, aunque la gravedad de las acciones no justifique una medida de protección (Rodrigo, Máiquez, Martín y Byrne, 2008).

El estudio hecho por Rodrigo et al., (2008) y Rodrigo, Martín, Cabrera y Máiquez (2009), muestra los principales factores de riesgo que impiden a estas familias cubrir las necesidades evolutivas y educativas de sus miembros. La característica central es el alto nivel de estrés familiar, deficiencias en cuanto a recursos y habilidades, para mantener la economía doméstica, y el contar con hogares en condiciones inadecuadas. Son en general ambientes disfuncionales que no favorecen la estimulación. Además, los adultos tienen problemas de salud, se sienten incompetentes y cuentan con trayectorias personales complicadas y con un alto fracaso educativo.

Diversos autores han hecho referencia al significativo lugar que tiene el estrés en la etiología del maltrato infantil (Belsky, 1980; Belsky y Vondra, 1989). Éste parecería tener un importante papel en el deterioro de las familias, en particular de las multiproblemáticas y en alto riesgo psicosocial, así mismo tendría un papel relevante en el desenlace de la VD.

\section{Estrés, definición y clasificación}

Selye (1956), define el estrés como un conjunto de respuestas fisiológicas inespecíficas a estímulos físicos externos o internos.

Por su parte Lazarus y Folkman (1986), clasificaron las situaciones estresantes según su intensidad en:

- cataclismos

- sucesos vitales estresantes de considerable magnitud (muerte en la familia, divorcio, emigración)

- pequeñas contrariedades diarias

A su vez Sandín (2003), habla de:

- estrés crónico 
- $\quad$ estrés diario

- $\quad$ sucesos vitales

Los sucesos menores o microestresores se caracterizan por ser más frecuentes y menos relevantes que los sucesos mayores (Sandín, 2003). Rodrigo et al., (2008) refieren la existencia de tres niveles de estrés de acuerdo a su mayor o menor gravedad:

- $\quad$ estrés cotidiano

- estrés crónico, muy asociado entre otras cosas a situaciones de VD

- $\quad$ sucesos vitales negativos

Por otro lado Boss (2002), propone clasificar los estresores según su fuente, en: internos o externos, su duración: crónicos o agudos, la densidad: aislados o acumulativos y el tipo: voluntarios o involuntarios, normativos o no normativos, ambiguos o definidos.

Con respecto a los estresores normativos (ocurren en la mayoría de las familias) los no normativos (no ocurren en todas las familias y son inesperados). Boss (2002), señala que ambas situaciones tienen un significativo potencial de generación de estrés familiar y Musitu y Cava (2001), refieren que ambas situaciones producen cambios en el funcionamiento familiar. Por su parte Barudy y Marquebreucq (2006), entienden que el estrés familiar es la tensión creada por circunstancias del entorno o por vicisitudes propias de la familia, que amenazan sus funciones, su bienestar y su existencia.

\section{Estrés familiar y violencia doméstica}

Las familias, como sistema, evolucionan a través de ciertas etapas que son bastante universales y se las denomina normativas (Carter y McGoldrick, 1989). Durante el proceso atraviesan períodos de equilibrio y adaptación y períodos de desequilibrio y cambio. Además durante el ciclo vital también nos encontramos con situaciones estresantes no normativas (Musitu y Cava, 2001), como ser, separaciones, divorcios, muerte prematura, enfermedad crónica, jubilaciones anticipadas, etc (Ochoa de Alda, 1995). Así mismo se presentan hechos o situaciones relevantes que producen cambios, perturbaciones, o crisis en el recorrido, que requieren modificaciones y adaptaciones con el fin de restablecer el funcionamiento por parte del individuo y la familia (Herrera, 2008). En este sentido Minuchin (1974), señala tres características que definen a una familia como normal: la familia es un sistema abierto y en proceso de transformación; la familia transita por diversas etapas teniendo que reestructurarse en cada una de ellas; y la familia deberá adaptarse a las nuevas circunstancias.

¿Cómo afectan las perturbaciones o crisis al desarrollo familiar? Ceberio (2013), manifiesta que las crisis generan un momento de alta inestabilidad en el sistema familiar. Otros autores, hablan de la importancia que tienen las crisis en el transcurso del ciclo vital como generadoras de tensión y estrés familiar, (Barudy, 1998; Boss, 2002) o como promotoras del cambio. Estos cambios se producen por problemas en el sistema familiar, ante un aumento de la tensión (Ceberio, 2013). En este punto y siguiendo a Boss (2002), cabe consignar que el estrés es una variable constante, que perturba el equilibrio familiar, mientras que la crisis es una gran presión que resulta abrumadora. El estrés se desencadena como consecuencia de la aparición de algún suceso o hecho en la familia (que amenaza sus funciones, su bienestar o su existencia) que interrumpe el desarrollo del ciclo vital y requiere de la familia adaptarse a las nuevas circunstancias (Barudy y Marquebreucq, 2006; Musitu y Cava, 2001).

Lo que define una situación como estresante, es el hecho de que se trata de un suceso que obliga a realizar cambios, a reestructurar el funcionamiento familiar. La clave es el modo en que la familia maneja esta situación, como la percibe, su capacidad de adaptación para realizar los cambios necesarios (Musitu y Cava, 2001).

\section{Riesgo, estrés y violencia doméstica}

La respuesta de estrés en sí misma no es perjudicial. Ha sido y es muy útil ante situaciones de amenaza. El problema se presenta cuando la reacción se da en forma frecuente, intensa o duradera. Esto mismo puede producir un deterioro de los recursos de la persona o de la familia, promoviendo diversos problemas o patologías asociadas al estrés (Crespo y Labrador, 2010). En definitiva, el estrés familiar actúa perturbando el equilibrio y es una fuerza que presiona la estructura familiar. Cuando además se trata de familias en las que acontecen situaciones de VD, se incrementa el riesgo.

Es en estos momentos en los que se potencializa el riesgo de que la estructura familiar sufra un franco deterioro. Una muestra de ello lo es la violencia hacia las mujeres y NNA. Según Gracia, Musitu, y García (1994), el maltrato infantil es la forma de mostrar la insuficiencia 
de recursos o habilidades que demostraron tener los adultos para afrontar y sobreponerse a situaciones con un alto nivel de estrés. Tal vez como consecuencia de un ajuste psicológico y social parental deficiente (recursos o habilidades), no pueden desarrollar comportamientos adaptativos.

Gracia et al. (1994), concluyen en su investigación que los padres que maltratan a sus hijos, en relación a los que no lo hacen, padecen niveles más altos de estrés, presentan una mayor presencia de síntomas y se encuentran aislados socialmente.

Esto no significa que el estrés sea la causa directa ni la única de la VD, ya que ésta es producto de la multicausalidad, así como tampoco la VD y el estrés son patrimonio de las familias en riesgo psicosocial.

\section{Respuesta Violenta}

¿Por qué es entonces que algunos adultos responden a ese incremento en la conflictiva, en la acumulación de tensión, en forma violenta hacia los NNA a su cargo? Tal vez la respuesta se encuentre condicionada por la propia historia personal de los padres, los valores reinantes en la sociedad, en la subcultura o en la comunidad, etc (López-Torrecillas, 2009), a los mínimos recursos económicos y sociales con los que cuentan algunos padres y madres, a su falta de competencias parentales (Barudy y Dantagnan, 2010). Bringiotti (2000), refiere factores de riesgo o predisponentes, entre ellos, malos tratos en su propia historia infantil, baja tolerancia a la frustración y al estrés, expectativas inadecuadas en relación a las capacidades de los hijos, falta de empatía, etc. Por su parte Garbarino y Eckenrode (1999), manifiestan como factores amenazantes, la utilización de sustancias tóxicas por parte de los padres, problemas psiquiátricos, cognitivos, entre otros.

Se ha visto que cuando el ajuste psicológico y social de los adultos referentes de los NNA es insuficiente, el riesgo de que el clima familiar se deteriore aumenta. Una evidencia de esto es la irrupción de la VD. Al parecer una constante en los adultos maltratantes es el malestar psicológico generalizado (Milner, 1990). Se ha considerado desde el comienzo de las investigaciones de maltrato infantil, que los padres y madres maltratadores padecen de sentimientos de inadecuación y baja autoestima (Anderson y Lauderdale, 1982). En muchas oportunidades al surgir una crisis en el sistema familiar y ante la falta de recursos materiales y/o psicosociales, puede aumentar la tensión familiar, corriendo un serio riesgo los más vulnerables (Barudy, 1998). La VD genera situaciones altamente estresantes, y conlleva un deterioro, psicosocial y físico tanto en las mujeres como en los NNA (Villavicencio y Sebastián, 1999). El hecho de estar expuesto a VD produce graves consecuencias en NNA (Patró y Limiñana, 2005).

\section{Estrés, violencia doméstica y psicopatología}

Como manifiesta Lazarus (2000), si existe un cuadro de estrés siempre están presentes las emociones. Hay una importante interdependencia entre el estrés y las emociones, ambos estados afectan el bienestar psicológico, el funcionamiento y la salud física de las personas. Actualmente es un hecho constatado que el estrés, las emociones y la salud mantienen entre sí una estrecha vinculación (Jiménez-Torres, Martínez, Miró y Sánchez, 2012).

La depresión es una de las emociones que se puede producir como consecuencia del estrés, aunque Lazarus (2000), plantea que también se presentan: ira, envidia, celos, ansiedad, temor, culpa, vergüenza, alivio, esperanza, tristeza que son las emociones estresantes y felicidad, orgullo, amor, gratitud y compasión, las emociones de tono positivo y las condiciones relacionales que las rodea.

Tal como surge de la investigación llevada a cabo por Herrero (como se citó en Gracia, Herrero y Musitu, 2002), en el caso de personas que experimenten un elevado número de eventos vitales estresantes, los mismos se asocian a un aumento de estados depresivos. En el estudio llevado adelante por el investigador con una muestra de 1200 sujetos, manifiesta que las personas que padecen un elevado número de eventos vitales estresantes presentan una devaluación de su ánimo, la media de depresión es mayor en estos sujetos. En esa misma investigación el autor observó el efecto del estrés sobre la autoestima. Los resultados indican una importante disminución de la autoestima como producto de niveles elevados de estrés seis meses antes. A su vez Gracia, Herrero y Musitu (1995), manifiestan que de contar el sujeto con una elevada autoestima, eso le anima a participar en un mundo de relaciones recíprocas, dando y recibiendo ayuda. Por otro lado Cuervo (2010), nos refiere que los problemas emocio- 
nales en los adultos, como por ej. la depresión, influyen en los NNA, transmitiéndoles modelos inadecuados o prácticas negativas para su desarrollo. La depresión trae aparejada la pérdida de interés o placer en actividades diarias, trastornos del sueño, sentimientos de infravaloración, falta de energía, dificultades a nivel del pensamiento y atencionales, tendencia al llanto, desesperanza e incluso ideación suicida (American Psychiatric Association [APA], 2001).

La depresión es un síntoma que suele aparecer con frecuencia en las víctimas de VD (Echeburúa, Corral y Amor, 1998). Por otra parte Paz, Labrador, Arinero y Crespo (2004), señalan en su investigación que la sintomatología por (TEPT, Trastorno por Estrés Postraumático), y la comorbilidad por depresión, alcanzan un alto porcentaje en las víctimas de VD. Así mismo, Patró, Corbalán y Limiñana (2007), manifiestan que los síntomas depresivos aumentan en la medida que las expresiones de violencia física, psicológica y sexual son más importantes, hay un incremento de estresores no relacionados con el maltrato y cuentan con un menor nivel de percepción de apoyo social. A su vez, Labrador, Fernández-Velasco y Rincón (2010), refieren que el TEPT es el conjunto de síntomas que define mejor a las mujeres víctimas de VD, aunque en la investigación que llevaron a cabo la depresión fue el síntoma más frecuente; también relevaron ansiedad, riesgos de suicidio y baja autoestima. De la misma forma, Echeburúa, de Corral, Amor, Sarasua y Zubizarreta, (1997), señalan en su estudio la alta incidencia de TEPT (Aguirre et al., 2010), ansiedad y depresión, en mujeres víctimas de violencia. Matud (2004), relata que las mujeres que han sido maltratadas sufren síntomas de depresión grave, ansiedad, insomnio y síntomas somáticos, baja autoestima y bajo apoyo social.

\section{Estrés, violencia doméstica y factores amortiguadores}

Ante la presencia de situaciones de VD la red familiar y social juegan un papel de vital importancia, tanto para la prevención, la detección o durante el proceso de reparación del daño y la restitución de los derechos vulnerados. El abordaje de NNA en muchas ocasiones está directamente relacionado al abordaje de su entorno familiar inmediato y a su vez, al abordaje del sistema comunitario dónde se inserta el núcleo familiar, con especial referencia en los centros educativos. De esta forma los procesos tanto de cambio como de salida, se producen en tanto se intervenga en todos los microsistemas y en su interrelación.

Máxime en situaciones de maltrato y abuso sexual, ya que la víctima NNA requiere tanto en el momento de la revelación de las situaciones de violencia, como posteriormente en el proceso de recuperación, del apoyo de adultos protectores significativos (Lichtenberg, 2008). Contar entonces con esa red, ejerce un efecto positivo en la autoestima de la persona, así como también en la capacidad de afrontar hechos difíciles o traumáticos (Lin y Ensel, 1989; Musitu, Buelga, Lila y Cava, 2001). Palomar y Cienfuegos (2007), manifiestan que las personas o instituciones capaces de brindar ayuda conforman lo que se denomina "redes sociales" y es a través de ellas que la familia recibe los recursos psicológicos y materiales que necesita.

Según lo señalado por Garbarino y Kostelny (1992), no tenemos que perder de vista que el maltrato es un síntoma de problemas, no sólo de carácter individual o familiar, sino también a nivel comunitario y de vecindad. Es un indicador no sólo psicológico sino también social. Según Lin y Ensel (1989), el apoyo social es el proceso por el cual los recursos sociales, comunidad, redes sociales y relaciones íntimas, permiten satisfacer necesidades, instrumentales y expresivas, en situaciones cotidianas y de crisis. Dentro de las funciones del apoyo social se encuentran: el apoyo emocional; el apoyo instrumental y el apoyo informacional (Gracia et al., 2002).

En tanto Pearlin (1989), manifiesta que el apoyo social no debe confundirse con las redes sociales. Las redes son consideradas como la totalidad de los recursos sociales potencialmente existentes a disposición de un individuo, el apoyo social representa los recursos reales que utiliza para hacer frente a las diversas vicisitudes de la vida.

En este sentido el apoyo social puede funcionar como amortiguador, moderador entre el ajuste psicológico y los estresores, por lo tanto altos niveles de apoyo minimizan y protegen de los efectos adversos que puedan generar los eventos estresantes (Gracia et al., 1994). Además, estos autores concluyen que cuando no hay suficientes y adecuados recursos parentales para sobrellevar altos niveles de estrés (Barudy, 1998), en una situación de aislamiento social y sin apoyo y ante la presencia de la frustración, 
rabia y desajuste psicológico, se potencia la posibilidad de que surjan situaciones de VD.

El apoyo social aparece como el recurso social de mayor importancia para la superación de las adversidades producidas por los eventos vitales estresantes. Las víctimas de este flagelo, que no cuenten con el apoyo social o familiar, presentan una mayor gravedad psicopatológica (Amor, Echeburúa, De Corral, Zubizarreta y Sarasua, 2002). Se da una situación paradojal en relación al apoyo social ya que es visto como el recurso social de mayor importancia para la superación de las adversidades producidas por los eventos vitales estresantes, sin embargo la ocurrencia de eventos vitales estresantes puede lograr la disminución de los apoyos sociales disponibles (Gracia et al., 2002).

Ha sido ampliamente estudiado el apoyo social en relación a la prevención a largo plazo de los efectos físicos y psicológicos, posteriores al evento traumático. Herrero (como se citó en Gracia et al. 2002), manifiesta que determinados eventos acaecidos en lo social, producen fuertes consecuencias en la salud mental de las personas. Esta situación se vivencia más claramente en situaciones de alto estrés. En estos casos el efecto que proporciona el apoyo social en la salud, es de carácter amortiguador o "buffer". El efecto amortiguador del apoyo social ha sido investigado en múltiples estudios (Gracia, 2011). Por esta razón, el apoyo aparece como un recurso de afrontamiento ante las situaciones estresantes. Podríamos concluir diciendo que el apoyo social refleja la transmisión de afectos de las personas próximas y, además, posibilita que el individuo se considere junto con otros, parte de un mundo social (Herrero, 2004).

\section{Metodología}

\section{Objetivo}

Identificar y cuantificar los eventos vitales estresantes y la percepción de estrés de los adultos referentes de niños, niñas y adolescentes que han vivido situaciones de violencia doméstica.

\section{Método}

El estudio de carácter descriptivo se enmarca en un diseño de investigaciónacción. Para León y Montero (2003), ésta representa el estudio de un contexto social donde mediante un proceso de investigación con pasos 'en espiral', se investiga al mismo tiempo que se interviene.

\section{Participantes}

En el presente estudio se analiza una muestra de 60 adultos referentes (padres, madres, tenedores, etc) de NNA. Las edades de los referentes se encuentran comprendidas entre 25 y 55 años y la media de rango se encuentra entre los 30 y 43 años.

El $11,7 \%$ de los referentes son hombres y el $88,3 \%$ mujeres. Los criterios de admisión para este estudio han sido que el adulto referente asista junto a los NNA voluntariamente y se encuentren participando activamente del plan de acción individual y/o familiar, que fuera diseñado por el equipo interdisciplinario. A efectos de homogeneizar la muestra, el único criterio de exclusión ha sido la presencia de trastorno mental o enfermedad orgánica grave.

\section{Instrumentos}

El instrumento utilizado para la evaluación de la percepción fue el Cuestionario de Estrés Percibido -CEP- (Cohen, Kamarck y Mermelstein, 1983; Gracia et al., 2002). Ha demostrado una adecuada validez discriminativa y un alfa de Cronbach, 0,83. Este cuestionario identifica el nivel de percepción de estrés que refieren los sujetos.

El segundo instrumento utilizado fue el Listado de Eventos Vitales Estresantes - LEE- de Gracia et al. (2002). Es un listado sobre eventos vitales que, por tal condición, no se manejan los parámetros de la psicometría clásica. El instrumento combina, la información procedente de situaciones objetivas que producen estrés y la interpretación que el individuo realiza de esas situaciones.

\section{Procedimiento}

Seleccionados los sujetos que iban a participar, se comienza con la toma de los cuestionarios de forma individual. Se les explicó a los participantes en qué consistía el estudio que íbamos a llevar a cabo. A continuación se les aclaró sobre la confidencialidad de los datos y la utilización que se iba a hacer de los mismos. Tras estas instrucciones los participantes completaron el cuestionario. 
El tiempo máximo para completarlos no superó los 15 minutos.

\section{Resultados}

En cuanto al CEP (Cohen, Kamarck y Mermelstein; Gracia et al., 2002), pasaremos a detallar los resultados que hemos entendido más significativos. El $27 \%$ de los adultos refieren "muy a menudo y bastante a menudo" y un $39 \%$ "a veces", que son incapaces de controlar las cosas más importantes de su vida. El 53\% "muy a menudo y bastante a menudo" y un 30\% "a veces", se han sentido tensos y nerviosos. El $15 \%$ "nunca y casi nunca" y un $36 \%$ "a veces", sintieron que se ha enfrentado de forma adecuada a los cambios que se producían en su vida. Un $44 \%$ "muy a menudo y bastante a menudo" y un $29 \%$ "a veces", se ha encontrado con que no podía con todas las cosas que debiera. Un $42 \%$ "muy a menudo y bastante a menudo" y un $21 \%$ "a veces", ha pensado que estaba harto de todo. Un $41 \%$ "muy a menudo y bastante a menudo" y un $25 \%$ "a veces", se han sentido furiosos porque las cosas que le sucedían escapaban a su control. Un 10\% "nunca o casi nunca" y un $31 \%$ "a veces", no se ha sentido capaz de controlar la forma en que distribuye su tiempo. Un 36\% "bastante a menudo y muy a menudo" y un $39 \%$ "a veces", ha sentido que las dificultades se le acumulaban sin poder solucionarlas (ver Gráfico 1).

La puntuación media del CEP (Cohen et al., 1983; Gracia et al., 2002), para la población general, se sitúa en torno a los 36 puntos, en tanto el personal hospitalario alcanza una puntuación de 43 puntos. En este sentido, nuestros resultados arrojan que un $56,6 \%$ de los adultos han puntuado 43 puntos y más, mientras que un $43,3 \%$ ha puntuado menos de 43 puntos.

El cuestionario además cuenta con dos puntuaciones separadas: a) percepción de falta de control y b) sentimientos de control. En a) percepción de falta de control, "bastante a menudo y muy a menudo" alcanza un $45 \%$, mientras que la variable "a veces" alcanza un $30 \%$. En cuanto a b) sentimientos de control, "nunca y casi nunca" alcanza un $11 \%$, mientras que la variable "a veces" llega a un $33 \%$.

En cuanto al cuestionario LEE (Gracia et al., 2002), tenemos que un $45,7 \%$ de los adultos refieren un incremento del número de problemas o asuntos sin resolver; un $40 \%$ manifiesta haber pasado por un incremento de los conflictos de los hijos/hermanos de la familia; un $37,2 \%$ manifiesta no haber podido hacer frente a los gastos o problemas económicos similares; un $33,8 \%$ indica que un miembro de la familia estuvo sin trabajo durante un prolongado período (despidos, permisos, huelgas, etc); un 32,2 contesta haber vivido la ruptura de los lazos afectivos íntimos o muy próximos; un $30,5 \%$ contesta haber vivido dificultades graves, con personas que están bajo su supervisión o responsabilidad; un $28,8 \%$ ha manifestado tener dificultades para educar a los hijos adolescentes; un $23,7 \%$ ha manifestado tener un incremento en los conflictos con la pareja. Se realizó

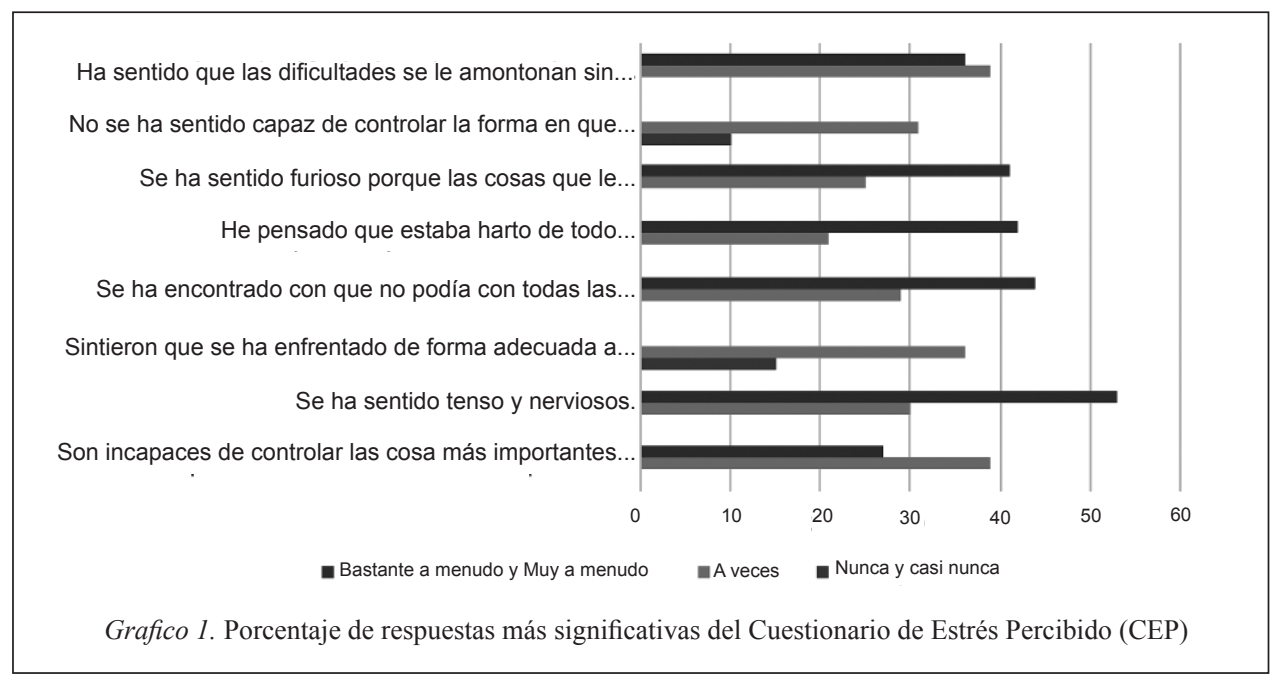


una categorización de los ítems que obtuvieron mayor puntuación de acuerdo a la temática que engloban. Tenemos entonces, que cinco de los ítems seleccionados por los adultos, refieren a dificultades vinculares/relacionales, dos a dificultades económicas/laborales y una a dificultades por problemas generales/asuntos sin resolver (ver Tabla 1). En este sentido nos parece relevante que las principales dificultades de los referentes, se encuentren relacionadas a problemas vinculares y/o económicos, áreas que probablemente afecten la emocionalidad de

Tabla 1. Distribución de adultos según categorizacion realizada en base a 8 items

\begin{tabular}{llcc}
\hline \multicolumn{1}{c}{ CUESTIONARIO LEE } & $\%$ & \multicolumn{1}{c}{ CATEGORÍA } \\
\hline 1 & $\begin{array}{l}\text { Refieren un incremento del } \\
\text { número de problemas o asuntos } \\
\text { sin resolver }\end{array}$ & 45,7 & $\begin{array}{c}\text { Dificultades por problemas } \\
\text { generales y asuntos sin resolver }\end{array}$ \\
2 & $\begin{array}{l}\text { Manifiesta haber pasado por un } \\
\text { incremento de los conflictos de } \\
\text { los hijos/hermanos de la familia }\end{array}$ & 40 & Dificultades vinculares/relacionales \\
3 & $\begin{array}{l}\text { Manifiesta no haber podido } \\
\text { hacer frente a los gastos o } \\
\text { problemas económicos similares }\end{array}$ & 37,2 & Dificultades económicas/laborales \\
4 & $\begin{array}{l}\text { Indica que un miembro de la } \\
\text { familia estuvo sin trabajo durante } \\
\text { un prolongado período (despidos, } \\
\text { permisos, huelgas, etc.) }\end{array}$ & 33.8 & Dificultades económicas/laborales \\
5 & $\begin{array}{l}\text { Contesta haber vivido la ruptura } \\
\text { de los lazos afectivos íntimos o } \\
\text { muy próximos }\end{array}$ & 32,2 & Dificultades vinculares/relacionales \\
6 & $\begin{array}{l}\text { Contesta haber vivido } \\
\text { dificultades graves, con } \\
\text { personas que están bajo su } \\
\text { supervisión o responsabilidad }\end{array}$ & 30.5 & Dificultades vinculares/relacionales \\
7 & $\begin{array}{l}\text { Ha manifestado tener } \\
\text { dificultades para educar a los } \\
\text { hijos adolescentes }\end{array}$ \\
8 & $\begin{array}{l}\text { Ha manifestado tener un } \\
\text { incremento en los conflictos con } \\
\text { la pareja }\end{array}$ & 28,8 & Dificultades vinculares/relacionales \\
\hline
\end{tabular}

estos adultos, sin dejar de mencionar y destacar, que el ítem que alcanzó el mayor puntaje es el que tiene relación con el incremento del número de problemas o asuntos sin resolver.

El $59,3 \%$ del total de referentes manifestaron haber experimentado en los últimos seis meses, menos de 5 eventos vitales estresantes, el 30,5\% marcó entre 5 y 10 sucesos y un 10,2 selecciono más de 10 (ver Gráfico 2). Siguiendo a McCubbin y Patterson (1983), la acumulación de eventos estresores es lo que determina el nivel de estrés familiar.

\section{Discusión}

El presente estudio se realizó con una población de 60 adultos referentes de NNA víctimas de violencia doméstica. El estudio es una experiencia piloto que intenta caracterizar a la población con la cual se trabaja y alcanzar mejores estrategias de intervención con las familias.

Algunos de los datos que resultan particularmente interesantes son que el $88,3 \%$ de las personas referentes que completaron los cuestionarios fueran mujeres. No encontramos diferencias significativas en el impacto de los eventos vitales estresantes así como de la percepción de estrés en función del sexo, observando especialmente la poca amplitud de la muestra y el sego de la misma. El 78,3\% son madres de NNA y el 9,9\% son abuelas, compa-

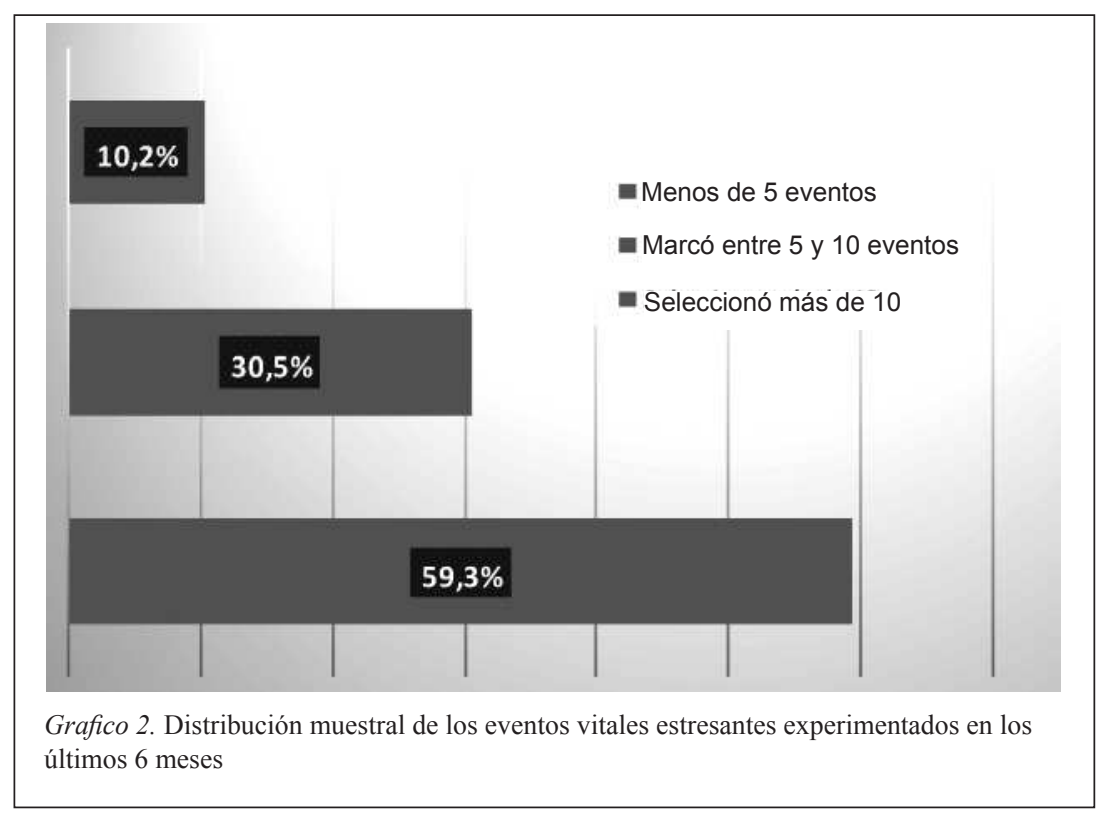


ñeras o esposas de los padres. Que la mayoría de las referentes sean mujeres, le agrega un grado de complejidad a las situaciones aun no siendo hogares monoparentales. La VD se encuentra presente en varías de las historias personales de las referentes mujeres de estos NNA, ya sea en la infancia/adolescencia, en sus familias de origen o en la adultez. La VD puede ser en sí misma un evento estresante. El tiempo de exposición, el tipo de violencia, la acumulación de eventos vitales estresantes, así como la percepción que ellas pueden tener acerca de la falta de control sobre éstos eventos, pueden llevarlas a que dejen de buscar una solución o tardar hasta 10 años de media en buscar una ayuda dada las consecuencias e implicancias a nivel personal, familiar y social (Labrador, Fernández-Velasco y Rincón, 2006). No obstante entre los distintos tipos de eventos vitales estresantes se producen solapamientos e interacciones. Es así que, además de las posibles situaciones crónicas mantenidas pueden sufrir eventos estresantes cotidianos afectando sus roles y funciones parentales entre otros aspectos. Es más, algunas vivencian una escalada de eventos o sucesiones de muchos de ellos que generan a su vez otros eventos también estresantes (Crespo y Labrador, 2010). Por ejemplo, el alejamiento del agresor conlleva además de una ruptura de pareja, otras situaciones que pueden también ser estresantes, como el enfrentar un proceso judicial, futuras agresiones y/o riesgo de vida, crianza y cuidado de sus hijos, perdida de actividades cotidianas, mudanza, presencia en los distintos servicios de ayuda para ellas, como así también para sus hijos/as, pérdida de apoyo social, pérdida de ingresos como sostén y dificultades económicas.

En este sentido debemos tener en cuenta la precarización laboral y económica que padecen muchas mujeres por una cuestión de género (Mazzei, 2013; Martínez, 2006), que además cuentan con hijos a cargo, altos niveles de estrés, con una probabilidad cierta de haber sufrido VD y en muchas situaciones, con un marcado deterioro emocional. El World Economic Forum nos refleja la brecha existente entre hombres y mujeres, principalmente en cuatro áreas trascendentes: participación económica y oportunidades; poder político; éxito educacional; y salud y supervivencia, y concluyen que "la desigualdad basada en el género es un fenómeno que afecta a la mayoría de los grupos de las diferentes culturas, religiones, naciones y niveles económicos" (World Economic Forum [WEF], 2007, p. 3).

En relación a lo visto a lo largo del estudio, podríamos inferir que, a partir de la alta incidencia en la percepción de estrés y de una elevada cantidad de eventos vitales estresantes vividos por los referentes adultos, habría probabilidades de que estos referentes padecieran problemas: gástricos, cardiovasculares, asma, consumo de alcohol, drogas, trastorno de ansiedad, depresión, problemas de relacionamiento social o familiar (Crespo y Labrador, 2010). Así mismo sería posible que atravesaran por situaciones de VD (Belsky, 1980; Belsky y Vondra, 1989; Gracia et al., 1994) o de que a partir de esta situación padecieran alguna psicopatología (Aguirre et al., 2010; Barudy, 1998; Echeburúa et al., 1998; Echeburúa et al.,1997; Labrador et al., 2010; Matud, 2004; Patró et al., 2007; Paz et al., 2004), o de que al existir ya dichas violencias, no pudieran proteger a los NNA adecuadamente, teniendo en cuenta la declinación física, cognitiva y social de las personas que sufren situaciones de estrés (Villavicencio y Sebastián, 1999) y al bajo nivel de apoyo social con el que cuentan las víctimas de VD. El apoyo social es un recurso de vital importancia para contrarrestar y superar las situaciones estresantes. Las víctimas de VD, que no tienen el apoyo social indispensable, sufren de mayores consecuencias psicopatológicas (Amor et al., 2002), TEPT, depresión, ansiedad, etc.

Las experiencias recabadas por los profesionales de la organización, sumadas a los resultados obtenidos en los cuestionarios, nos llevan a pensar que efectivamente ante la existencia de una alta incidencia en la percepción de estrés y de una elevada cantidad de eventos vitales estresantes, los integrantes del núcleo familiar padezcan un alto nivel de estrés. Esto desvía tal vez, la atención de los adultos hacia otros temas trascendentes y pueden en ciertas ocasiones volverse insensibles ante características de sus hijos y ante sus reacciones, toman decisiones de forma rígida y automática y no prestan atención a las consecuencias que acarrean las acciones educativas que toman sobre sus hijos (Rodrigo, 2009), menoscabando, creemos nosotros, en algunas situaciones la capacidad de cuidar y proteger. Esta disminución de la capacidad de cuidar y proteger, se denota con claridad ante la repetición de las situaciones maltratantes que sufren los niños y adolescentes y en la insuficiente e inadecuada 
respuesta de los adultos (López-Soler, 2008), que han sufrido un deterioro de su emocionalidad. La recurrencia de las situaciones de violencia vividas por los NNA, es un dato que compartimos con otros actores, ya que según la base de datos de la ONG, un $89 \%$ de los NNA, han vivido situaciones recurrentes de VD. De la misma forma el Informe de Gestión, (Sistema Integral de Protección a la Infancia y a la Adolescencia contra la Violencia [SIPIAV], 2012), refiere una alta tasa de recurrencia $92 \%$ y una tasa de cronicidad de $81 \%$ en los NNA, asistidos por el sistema. Cabe consignar que la situación emocional y de salud mental de los referentes encuestados no fue relevada, así como tampoco el nivel de apoyo social con el que cuentan. A partir de este estudio, nos encontramos con la necesidad de promover nuevas investigaciones, que puedan darnos mayores elementos sobre los puntos reseñados.

Queremos advertir sobre algunas limitaciones del presente estudio referentes a la extensión y limitación de la muestra y la obtención de datos mediante instrumentos autoadministrados, que hacen que los resultados hallados deban de ser interpretados con prudencia, de cara a la generalización en otras poblaciones.

\section{Consideración final}

En Uruguay no existen estudios sobre el impacto del estrés en adultos referentes de NNA que han sufrido situaciones de VD. Los datos de esta experiencia son los primeros que se dan a conocer, pretendiendo ser una aproximación a algunas de las características de la población adulta con la que trabajamos. Muy a menudo estos individuos tienen que enfrentar distintas situaciones problemáticas con escasos recursos y habilidades. Ahora bien, las inferencias que fuimos realizando, nos llevan a pensar que habría que diseñar nuevas formas de intervención, con un nivel de integralidad mucho mayor, logrando nuevos compromisos de los diversos actores intervinientes. Es así que se vuelve imprescindible una revisión y adecuación legislativa, como también se requieren políticas que lleven adelante un plan integral, con los suficientes recursos económicos, materiales y humanos. Una política intersectorial e interinstitucional, que permita en paralelo resolver las dificultades que se les presentan a las familias en riesgo psicosocial, con altos niveles de estrés y con situaciones de VD, en los planos económico, laboral, de vivienda, de salud y educacional; en definitiva, que permita la restitución integral de los derechos de los NNA y sus referentes adultos.

A pesar de las limitaciones el estudio puede aportar orientaciones para la intervención psicológica y social de las familias, así como tal vez pueda utilizarse como recomendación para los organismos financiadores y responsables del diseño de las políticas públicas.

\section{Referencias}

Aguirre, P., Cova, F., Domarchi, Ma. P., Garrido, C., Mundaca, I., Rincón, P.,...Vidal, P. (2010). Estrés postraumático en mujeres víctimas de violencia doméstica. Revista Chilena de Neuro-Psiquiatría, 48 (2) 114-122

American Psychiatric Association (2001). DSM-IV TR. Manual diagnóstico y estadístico de los trastornos mentales. Barcelona: Masson.

Amor, P., Echeburúa, E., De Corral, P., Zubizarreta, I. y Sarasua, B. (2002). Repercusiones psicopatológicas de la violencia doméstica en la mujer en función de las circunstancias del maltrato. Revista Internacional de Psicología Clínica y de la Salud. Vol. 2 (2) 227-246

Anderson, S.C. y Lauderdale, M.L. (1982). Characteristics of abusive parents: a look of self-esteem. Child Abuse \& Neglect, 6, 285-293.

Barudy, J. (1998). El dolor invisible de la infancia, una lectura ecosistémica del maltrato infantil. España: Paidós

Barudy, J. y Marquebreucq, A. (2006). Hijas e hijos de madres resilientes. Traumas infantiles en situaciones extremas: violencia de género, guerra, genocidio, persecución y exilio. Barcelona: Gedisa

Barudy, J. y Dantagnan, M. (2010). Los desafíos invisibles de ser madre o padre. Manual de evaluación de las competencias y la resiliencia parental. Barcelona: Gedisa

Belsky, J. (1980). Child maltreatment: An ecological integration.American Psychologist, 35, 320-335.

Belsky, J. y Vondra, J. (1989). Lessons from child abuse: The determinants of parenting. En D. Cicchetti y V. Carlson (Eds.), Child maltreatment: Theory and research on the causes andconsequences of child abuse and neglect.(pp. 153-202) New York: Cambridge University Press.

Boss, P. (2002). Family stress management: A contextual approach. (2nd ed.). Thousand Oaks, CA: SAGE. doi: http://dx.doi.org/10.4135/9781452233895

Bringiotti, M. (2000). La escuela ante los niños maltratados. Buenos Aires: Paidós

Carter, E. y McGoldrick, M. (1989). The family life cycle. Aframework for family therapy. Nueva York: Brunner y Mazel.

Ceberio, M. R. (2013). El cielo puedeesperar.Argentina: Morata.

Crespo, M. y Labrador, F. (2010). Estrés. España: Síntesis

Cohen, S. Kamarck, T. \& Mermelstein, R. (1983). A global measure of perceived stress. Journal of Health and Social Behaviour, 24, 385-396

Cuervo, A. (2010). Pautas de crianza y desarrollo socioafectivo en la infancia. Perspectiva Psicológica 6 (1) 111-121. 
De los Campos, H., Solari, M. y González, M. (2008). Prácticas de Crianza y Resolución de Conflictos Familiares. Prevalencia del Maltrato intrafamiliar contra niñas, niños y adolescentes. Recuperado de http:// resourcecentre.savethechildren.se/sites/default/files/ documents/3653.pdf

Echeburúa, E., de Corral, P. y Amor, P. (1998) Perfiles diferenciales del trastorno de estrés postraumático en distintos tipos de víctimas. Análisis y modificación de la conducta, 24, (96) 527-555.

Echeburúa, E., de Corral, P., Amor, P., Sarasua, B. y Zubizarreta, I. (1997). Repercusiones Psicopatológicas de la Violencia Doméstica en la Mujer: Un estudio descriptivo. Revista de Psicopatología y Psicología Clínica, 2 (1) 7-19

Garbarino, J. \& Kostelny, K. (1992). Los malos tratos infantiles como problemas comunitarios. Anuario de Psicología , (53) 137-148

Garbarino, J. \& Eckenrode, J. (1999). Por qué las familias abusan de sus hijos. Barcelona: Granica

Gracia, E. (2011). Apoyo social e intervención social y comunitaria. En I. Fernández, F. Morales \& F. Molero (Coords) Psicología de la Intervención Comunitaria. (pp.129-172) España: Desclée De Brouwer

Gracia, E., Musitu, G. y García, F. (1994). Estrés, apoyo social y ajuste psicológico en padres que maltratan a sus hijos. Revista de Psicología Social, 9 (2) 193-205

Gracia, E., Herrero, J. y Musitu, G. (1995). El apoyo social. Barcelona: PPU.

Gracia, E., Herrero, J. y Musitu, G. (2002). Evaluación de recursos y estresores psicosociales en la comunidad. Madrid: Síntesis

Gómez, E., Muñoz, M. y Haz, A. (2007). Familias Multiproblemáticas y en Riesgo Social: Características e Intervención. Psikhe, 16, (2) 43-54

Herrera, P. (2008). El estrés familiar, su tratamiento en la psicología. Revista Cubana de Medicina General Integral, 24(3),1-8.

Herrero Olaizola, J. (2004). Redes sociales y apoyo social. En Musitu Ochoa G., Herrero Olaizola J., Cantera Espinosa L. y Montenegro Martínez M. Introducción a la Psicología Comunitaria. Barcelona: Ed. UCO

Jiménez-Torres, M., Martínez, M., Miró, E. y Sánchez, A. (2012). Relación entre estrés percibido y estado de ánimo negativo: diferencias según el estilo de afrontamiento. Anales de Psicología, 28 (1) 28-36

Labrador, F., Fernández-Velasco, R. y Rincón, P. (2010). Características psicopatológicas de mujeres víctimas de violencia de pareja. Psicothema, 22 (1) 99-105.

Labrador, F., Fernández-Velasco, R. y Rincón, P. (2006). Eficacia de un programa de intervención individual y breve para el trastorno por estrés postraumático en mujeres víctimas de violencia doméstica. International Journal of Clinical and Health Psychology, Vol. 6 (3) 527-547.

Lazarus, R. (2000). Estrés y Emoción, Manejo e implicaciones en nuestra salud. España: Desclée De Brouwer

Lazarus, R. S., \& Folkman, S. (1986). Estrés y procesos cognitivos. Barcelona: Martínez Roca.

León, O. y Montero, I. (2003). Métodos de Investigación en Psicología y Educación. Madrid: Mc Graw Hill

Ley 17514. Violencia Doméstica (2002).

Lichtenberg, P. (2008). Psicología de la Opresión, Guía para terapeutas y activistas. Chile: Cuatro Vientos
Lin, N. y Ensel, W. (1989). Life stress and health: Stressors and resources. American Sociological review, 54, 382-399.

Linares, J. L. (1997). Modelo sistémico y familia multiproblemática. En M. Coletti, y J. L. Linares, (comp) La intervención sistémica en los servicios sociales ante la familia multiproblemática. (pp. 23-43) Barcelona: Paidós Ibérica

López-Soler, C. (2008). Las reacciones postraumáticas en la infancia y adolescencia maltratada: el trauma complejo. Revista de psicopatología y psicología clínica, 13, (3) 159-174.

López-Torrecillas, J. (2009). Maltrato Infantil. Recuperado de http://www.uam.es/personal_pdi/psicologia/jlopezto/ Apuntes/Maltrato.pdf

Martínez, E. (2006). Introducción del enfoque de género en la orientación laboral. Recuperado de http://www. redacoge.org/empresas/redacoge/documentos/intervencion/Enfoquegenero.pdf

Matud, M.P. (2004). Impacto de la violencia doméstica en la salud de la mujer maltratada.Psicothema, 16 (3) 397-401

Mazzei, C. (2013). Producción y reproducción: la mujer y la división socio-sexual del trabajo. RUMBOS TS, VII, (8) 128-142.

McCubbin, H.I. y Patterson, J.M. (1983). The family stress process: the double ABCX model of adjustment and adaptation. In H. I McCubbin, M.B.Sussman, \& J. M. Patterson (eds.): Social stress and the family (pp. 7- 37). New York: Haworth.

Milner, J.S. (1990). Características familiares y del perpetrador en los casos de maltrato físico y abuso sexual infantil. Infancia y Sociedad, 2, 5-15

Minuchin, S. (1974). Familias y Terapia Familiar. Barcelona: Gedisa

Minuchin, P., Colapinto, J. y Minuchin, S. (2000). Pobreza, institución, familia. Argentina: Amorrortu

Musitu, G., Buelga, S., Lila, M. S. y Cava, M. J. (2001). Familia y Adolescencia. Madrid: Síntesis.

Musitu, G. y Cava, M.J. (2001). La familia y la educación. Barcelona: Octaedro

Navarro, I., Musitu, G. y Herrero, J. (2007). Familias y Problemas España: Sintesis

Observatorio Nacional sobre Violencia y Criminalidad (2012). Informe anual sobre violencia y criminalidad en todo el país año 2012. Recuperado de http:// medios.presidencia.gub.uy/jm_portal/2012/noticias/ NO_F172/OC_todo_2012.pdf

Ochoa de Alda, I. (1995). Enfoques en terapia familiar sistémica. Barcelona: Herder.

Pakman, M. (2006). Reducción del riesgo en familias múltiproblemáticas: la micropolítica de la justicia social en la asistencia en salud mental. Recuperado de http:// www.obelen.es/upload/343H.pdf

Palacios, J., Jiménez, J., Oliva, A. y Saldaña, D. (1998). Malos tratos a los niños en la familia. En Rodrigo, M.J. y Palacios, J. (Coords) Familia y Desarrollo Humano. (pp. 399-421) Madrid: Alianza Editorial

Palomar, J. y Cienfuegos, Y. (2007). Pobreza y Apoyo Social: Un estudio comparativo en tres niveles socioeconómicos. Revista Interamericana de Psicología, 41 (2) 177-188

Patró, R. y Limiñana, R. (2005). Víctimas de violencia familiar: Consecuencias psicológicas en hijos de mujeres maltratadas. Anales de Psicología, Vol. 21 (1) $11-17$ 
Patró, R., Corbalán, F. y Limiñana, R. (2007) Depresión en mujeres maltratadas: Relaciones con estilos de personalidad, variables contextuales y de la situación de violencia. Anales de Psicología, 23 (001) 118-124

Paz, P., Labrador, F., Arinero, M. y Crespo, M. (2004) Efectos psicopatológicos del maltrato doméstico. Avances en Psicología Latinoamericana, 22, 105-116

Pearlin, L.I. (1989). The sociological study of stress. Journal of Health and Social Behavior, Vol. 30 (3) 241-256.

Rodrigo, M.J. (2009). Una mirada integradora de la resiliencia parental: desde el contexto hasta la mente de las madres y padres en riesgo psicosocial. Psicología da Educacao 28, (1) 51-71

Rodrigo, M.J., Máiquez, M.L., Martín, J. y Byrne, S. (2008). Preservación Familiar Un enfoque positivo para la intervención con familias. Madrid: Pirámide

Rodrigo, M. J., Martín, J. C., Cabrera, E. y Máiquez, M. L. (2009). Las competencias parentales en contextos de riesgo psicosocial. Intervención Psicosocial, 18(2), 113-120.

Sandin, B. (2003). El estrés: un análisis basado en el papel de los factores sociales. Revista Internacional de Psicología Clínica y de la Salud, 3 (1) 141-157
Sanmartin, J.(2006). Violencia contra la mujer en las relaciones de pareja. Estadísticas y legislación. (II Informe Internacional). Recuperado de http:// www.observatorioviolencia.org/upload_images/File/ DOC1180979429_II\%20Informe\%20Internacional.pdf Selye, H. (1956). The stress of life. New York: McGrawHill. Sistema Integral de Protección a la Infancia y a la Adolescencia contra la Violencia. (2012). Modelo de atención integral. Violencia hacia niños, niñas y adolescentes. Recuperado de file:///C:/Users/cienciaspsi/ Downloads/11SIPIAV\%202012\%20MODELO\%20 ATENCION\%20INTEGRAL.pdf

Villavicencio, P. y Sebastián, J. (1999).Variables predictoras del ajuste psicológico en mujeres maltratadas desde un modelo de estrés. Psicología Conductual, 7 (3) 431-458

World Economic Forum (2007). The Global Gender Gap Report. Recuperado de http://www.weforum.org/ issues/globalgender-gap.

Para citar este artículo:

Capano, A. \& Pacheco, A. (2014). Estrés y violencia doméstica: un estudio en adultos referentes de niños, niñas y adolescentes. Ciencias Psicológicas VIII (1): 31- 42 